\title{
Erratum to: Design of a discrete algebraic robust differentiation FIR filter using an annihilator of the Z-transform; frequency response analysis and parameter tuning
}

\section{Fabien Courreges ${ }^{1}$}

Published online: 27 June 2015

(C) Springer Science+Business Media New York 2015

Erratum to: Numer Algor (2015) 68:867-901

DOI 10.1007/s11075-014-9875-3

Unfortunately, there is a misprint in the lines between Eq. 28 and 29.

In page 878, the line of text between equations (28) and (29) should be read "where $\forall i \in \mathbb{N}, i>a$," instead of "where $\forall i \in \mathbb{N}, i$ i $a$,".

Fabien Courreges

fabien.courreges@unilim.fr

1 Xlim Institute, Mechatronics team, University of Limoges, Limoges, France 